\title{
Comentarios y observaciones acerca de la diatomea Craspedostauros indubitabilis (Lange-Bertalot \& Genkal) E.J. Cox encontrada por primera vez en aguas marinas costeras de Chile
}

\section{Comments and observations on the diatom Craspedostauros indubitabilis (Lange- Bertalot \& Genkal) E.J. Cox found for the first time in marine coastal waters of Chile}

\author{
Patricio Rivera ${ }^{1}$, Fabiola Cruces ${ }^{1} \&$ Luc Ector $^{2}$ \\ ${ }^{1}$ Departamento de Botánica, Facultad de Ciencias Naturales y Oceanográficas, Universidad de Concepción, Casilla 160-C, \\ Concepción, Chile. \\ 2 Public Research Centre-Gabriel Lippmann, Department of Environment and Agro-Biotechnologies (EVA), Rue du Brill, 41, \\ L-4422 Belvaux, Grand-duchy of Luxembourg. \\ privera@udec.cl
}

\section{RESUMEN}

Especímenes de Craspedostauros indubitabilis (Lange-Bertalot \& Genkal) E.J. Cox provenientes de una muestra recolectada en Bahía Salada, Caldera, Chile $\left(27^{\circ} 38^{\prime} \mathrm{S}-71^{\circ} 00^{\prime} \mathrm{W}\right)$ fueron oxidados y examinados mediante técnicas de microscopía fotónica y electrónica. El género y la especie son señalados por primera vez para las aguas marinas costeras de Chile. Craspedostauros fue descrito por Cox en 1999 para reunir algunas diatomeas marinas birrafidales, ubicadas hasta esa fecha en los géneros Stauroneis Ehrenberg y Stauronella Mereschkowsky. El género puede ser reconocido por sus aréolas provistas de cribra, un estauro angosto, numerosas bandas en el cingulum y dos cloroplastos en forma de $\mathrm{H}$ con una peculiar disposición en la zona central. De acuerdo con el material chileno, Craspedostauros indubitabilis se caracteriza por poseer valvas linear-elípticas a lanceoladas con extremos generalmente redondeados, las estrías (22-30 en $10 \mu \mathrm{m})$ son paralelas en el centro de la valva y levemente convergentes cerca de los extremos, las aréolas adyacentes al rafe son de mayor tamaño que las restantes, cada aréola está ocluida por 3 a 13 poros, y un engrosamiento de sílice separa los extremos proximales internos del rafe. Se provee una diagnosis de este taxón basado en las características observadas en el material chileno. Las fotomicrografías que se presentan entregan información acerca de sus principales características morfológicas.

Palabras clave: Craspedostauros indubitabilis, Bacillariophyceae, morfología, taxonomía, distribución, Chile.

\begin{abstract}
Specimens of Craspedostauros indubitabilis (Lange-Bertalot \& Genkal) E.J. Cox from a sample taken in Bahía Salada, Caldera, Chile $\left(27^{\circ} 38^{\prime}\right.$ S $\left.-71^{\circ} 00^{\prime} \mathrm{W}\right)$ were acid cleaned and examined by light and electron microscopy. The genus and the species are reported for the first time from Chilean marine coastal waters. Craspedostauros was described in 1999 by Cox for some marine biraphid diatoms previously placed in Stauroneis Ehrenberg and Stauronella Mereschkowsky. The genus is recognized by its cribrate areolae, narrow stauros, numerous girdle bands and two chloroplasts fore and aft of the central cytoplasm. According to the Chilean material, Craspedostauros indubitabilis is characterized by its linear-elliptic to lanceolate valves usually with rounded ends, the striae $(22-30$ in $10 \mu \mathrm{m})$ are parallel in the valve centre and slightly convergent near the apices, the areolae adjacent to the raphe are much larger than over the rest of the valve, each areolae is occluded by 3 to 13 pores, and a knob of silica separates the central internal raphe endings. A diagnosis is here included for this taxon based on the characteristics observed in the Chilean material. The presented photomicrographs aim to provide information about the main morphological features.
\end{abstract}

KeYwords: Craspedostauros indubitabilis, Bacillariophyceae, morphology, taxonomy, distribution, Chile. 


\section{INTRODUCCIÓN}

El género Craspedostauros fue establecido por Cox (1999a) para incluir algunas especies de diatomeas birrafidales marinas previamente ubicadas en los géneros Stauroneis Ehrenberg y Stauronella Mereschkowsky. El nuevo género se caracteriza por poseer valvas contraídas al centro en vista conectival, numerosas bandas en el cingulum que llevan dos líneas de aréolas, que al igual de aquellas presentes en las valvas, exhiben una cribra de contorno cuadrangular a hexagonal. Además, se caracteriza por presentar una fascia más ancha que el estauro, por llevar una helictoglosa en cada uno de los extremos proximales internos del rafe, reemplazada en algunas especies por una única protuberancia de sílice, y por tener dos cloroplastos en forma de $\mathrm{H}$ por célula con una peculiar disposición en la zona central, cada uno con un pirenoide. En su publicación de 1999a, Cox también postuló que las características propias de las aréolas y los cloroplastos hacen que Craspedostauros presente más afinidades con las Mastogloiaceae que con las Naviculaceae. Los resultados de un análisis llevado a cabo con 21 especies de diatomeas naviculoides birrafidales, usando características del protoplasma y del frústulo, confirmaron la posición de Crapedostauros en las Mastogloiaceae (Cox \& Williams 2000).

Durante el desarrollo del proyecto FONDEF DO71-1017 "Biotecnología aplicada a la producción de alimentos y dietas balanceadas con la finalidad de mejorar el cultivo del Ostión del Norte", se recolectó una muestra de fitoplancton marino en Bahía Salada, Caldera, Chile. Cultivos unialgales de las diatomeas contenidas en dicha muestra permitieron reconocer a Craspedostauros indubitabilis (Lange-Bertalot \& Genkal) E.J. Cox, género y especie no señalados anteriormente para Chile. Esta especie fue descrita por Lange-Bertalot \& Genkal en 1999 como Stauronella indubitabilis a partir de material del Océano Ártico. El género Stauronella (Mereschkowsky 1901) está basado en Stauroneis constricta Ehrenberg (1843), una diatomea de agua dulce descrita para Chile. Por ello Cox (1999a) no lo reconoció como el nombre genérico de taxones similares que se desarrollan en ambientes marinos y creó para ellos el género Craspedostauros que, hasta la fecha, está compuesto por ocho especies.

En el presente trabajo se entrega una diagnosis de esta especie basada en las características observadas en el material chileno, y comentarios sobre sus semejanzas y diferencias con las restantes especies del género.

\section{MATERIAL Y MÉTODOS}

Craspedostauros indubitabilis fue encontrado en una muestra de fitoplancton costero recolectada en octubre de 2008 en Bahía Salada, Caldera (27 $38^{\prime}$ S $\left.-71^{\circ} 00^{\prime} \mathrm{W}\right)$, tomada en la columna de agua existente sobre el sistema de cultivo del ostión del norte (Argopecten purpuratus Lamarck). Células aisladas de estas muestras se cultivaron en el Laboratorio de Microalgas de la Universidad de Concepción en medio Walne + Si (Walne 1970) a una temperatura de $15 \pm 2{ }^{\circ} \mathrm{C}$, con un fotoperíodo de $16 \mathrm{~h}$ luz y $8 \mathrm{~h}$ de oscuridad y a 10-20 $\mu \mathrm{mol}$ de fotones por metro cuadrado por segundo. Luego de la eliminación de la materia orgánica según el método de Hasle \& Fryxell (1970), alrededor de 43 células fueron analizadas mediante técnicas de microscopía fotónica (LM, Zeiss Fotomicroscopio III), electrónica de barrido (SEM, Jeol JSM-6380LVY) y de transmisión (TEM, Jeol 1200 EX II). El material estudiado se encuentra depositado en la Colección Diatomológica de la Universidad de Concepción, Chile, bajo los números DIAT-CONC M-3347 y DIATCONC 7306, 7307. La terminología usada corresponde a la sugerida por Anonymous (1975), Ross et al. (1979) y Cox (1999a).

\section{RESULTADOS Y DISCUSIÓN}

Craspedostauros indubitabilis (Lange-Bertalot \& Genkal) E.J. Cox, Eur. J. Phycol. 34: 146. 1999.

Figs. 1-2.

Basiónimo: Stauronella indubitabilis Lange-Bertalot \& Genkal, Iconogr. Diatomol. 6: 100, pl. 36, figs. 1-8. 1999.

En vista conectival, los frústulos presentan un angostamiento en la parte central, los extremos valvares son redondeados, y el cingulum está formado por numerosas bandas abiertas (Fig. 1 A), provistas de una doble fila de aréolas, 28-30 en $10 \mu \mathrm{m}$, excepto al centro de la valva donde existe una sola (Fig. 1 B, flechas). Las valvas son fuertemente convexas, linear-elípticas a lanceoladas (Fig. 1 C-J), los extremos son redondeados, ocasionalmente cuneados (Fig. $1 \mathrm{H}$ ). Eje apical 16-27 $\mu \mathrm{m}$, eje transapical 4,4-7,6 $\mu \mathrm{m}$. El rafe es filiforme, más o menos derecho, con un rafe-sternum angosto (Fig. 1 E-J); externamente los extremos distales se curvan fuertemente en la misma dirección (Fig. 1-E-F), los extremos proximales son levemente ensanchados y curvados suavemente en la misma dirección, que es opuesta a la de los extremos terminales (Figs. 1 E-F, 2 A); internamente los extremos distales llevan una bien estructurada helictoglosa (Figs. 1G, 2 C) y los extremos proximales son filiformes, casi derechos, y entre ellos existe un engrosamiento de sílice (Figs. 1 G-J, 2 B). Las estrías son visibles con microscopía fotónica, 22-30 en $10 \mu \mathrm{m}$, y se orientan paralelas al centro de la valva y levemente convergentes hacia los extremos, en los cuales también hay algunas estrías (Fig. 2 C). Todas las aréolas (incluyendo aquéllas presentes en las bandas del cingulum) tienen un contorno cuadrangular a hexagonal y están ocluidas por una cribra externa estructurada por un número variable de poros (3-13), los cuales, en un mismo 
individuo, se pueden presentar de diferente forma con respecto a la disposición de los poros en el borde y en el centro de la cribra: 2/1, 4/1, 5/1, 6/1, 5/2,6/2, 7/2, 7/3, 9/4, siendo los más comunes 4/1 y 5/1 (Fig. 2 D). En esta especie las aréolas adyacentes al rafe son de mayor tamaño que las restantes (Figs. 1 E, 2 A). Con microscopía fotónica, el estauro es muy notorio, y más angosto que la fascia que lo acompaña hasta el margen valvar; es más grueso y más ancho en la parte central de la valva, luego se enangosta y adelgaza hacia los márgenes de la valva donde nuevamente se ensancha (Figs. 1 E-J, 2 A-B).

Características bastante estables de los individuos observados fueron la orientación y número de estrías sobre la valva, el mayor tamaño de las aréolas ubicadas al lado del rafe, la existencia de un estauro angosto y de una fascia ancha, las características relacionadas con las bandas del cingulum y del rafe, especialmente la presencia de una protuberancia entre los extremos centrales internos. Valvas con los márgenes angostados en la zona central fueron observadas solamente en material oxidado y montado en preparaciones permanentes. Estas observaciones, especialmente las llevadas a cabo con técnicas de microscopía electrónica, concuerdan con las realizadas por E.J. Cox (1988) en material reciente de Stauroneis constricta recolectado en Inglaterra y Sudáfrica. El número y distribución de los poros presentes en la cribra de las aréolas fue muy variable, y fue una de las características usadas por Cox (1999a) para diferenciar las especies de Craspedostauros. En una misma valva de $C$. indubitabilis se pueden presentar configuraciones diversas que comprenden de tres a trece poros (Fig. 2 D), variación que es menor en las restantes especies del género (Cox 1999a, b, Sabbe et al. 2003).
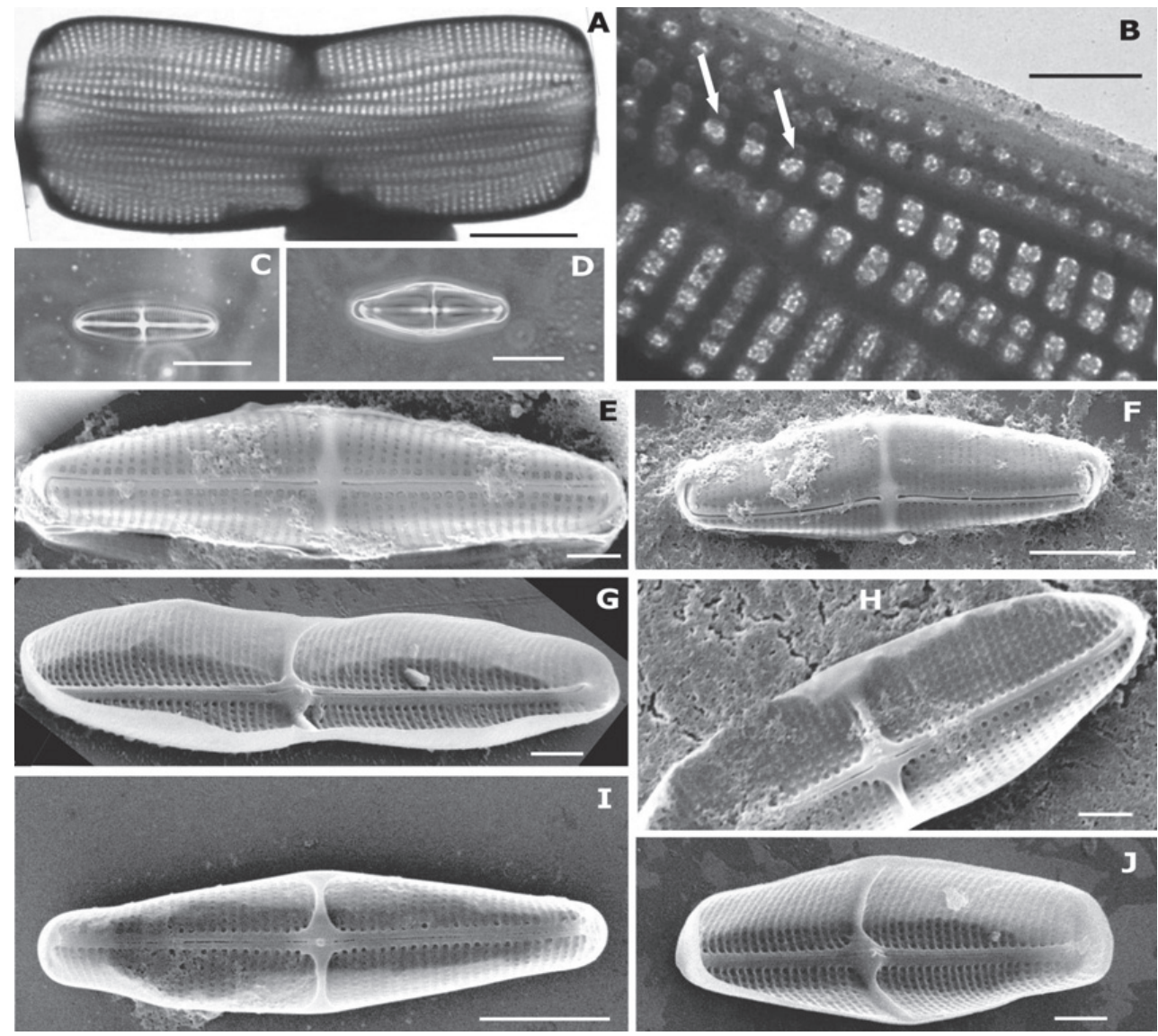

Figura 1. Craspedostauros indubitabilis. Figs. A-B TEM, Figs. C-D LM, Figs. E-J SEM. A. Frústulo en vista conectival, contraído al centro y con muchas bandas. B. Bandas con doble fila de aréolas cribradas, pero simple en la zona central (flechas). C. Valva linear-elíptica. D. Valva lanceolada. E-F. Vista externa de valvas mostrando las características del rafe. G. Valva contraída en la zona central; estauro y fascia notorios. H. Vista interna de una valva con extremos cuneado-redondeados. I. Protuberancia entre los extremos proximales del rafe. J. Estauro y fascia notorios. Escalas A, F, I $=5 \mu \mathrm{m}, \mathrm{B}=1 \mu \mathrm{m}, \mathrm{C}-\mathrm{D}=10 \mu \mathrm{m}, \mathrm{E}, \mathrm{G}-\mathrm{H}, \mathrm{J}=2 \mu \mathrm{m}$.

Figure 1. Craspedostauros indubitabilis. Figs. A-B TEM, Figs. C-D LM, Figs. E-J SEM. A. Girdle view of a cell centrally constricted and with many girdle bands. B. Girdle bands with double rows of cribrated areolae, except in the middle (arrows). C. Linear-elliptic valve. D. Lanceolate valve. E-F. External view of valves showing characteristics of the raphe. G. Valve centrally constricted; stauros and fascia. H. Internal view of valve with cuneate-rounded ends. I. Knob-like thickening between the proximal internal ends. J. Stauros and fascia obvious. Scale bars: A, F, I: $=5 \mu \mathrm{m}, \mathrm{B}=1 \mu \mathrm{m}, \mathrm{C}-\mathrm{D}=10 \mu \mathrm{m}, \mathrm{E}, \mathrm{G}-\mathrm{H}, \mathrm{J}=2 \mu \mathrm{m}$. 


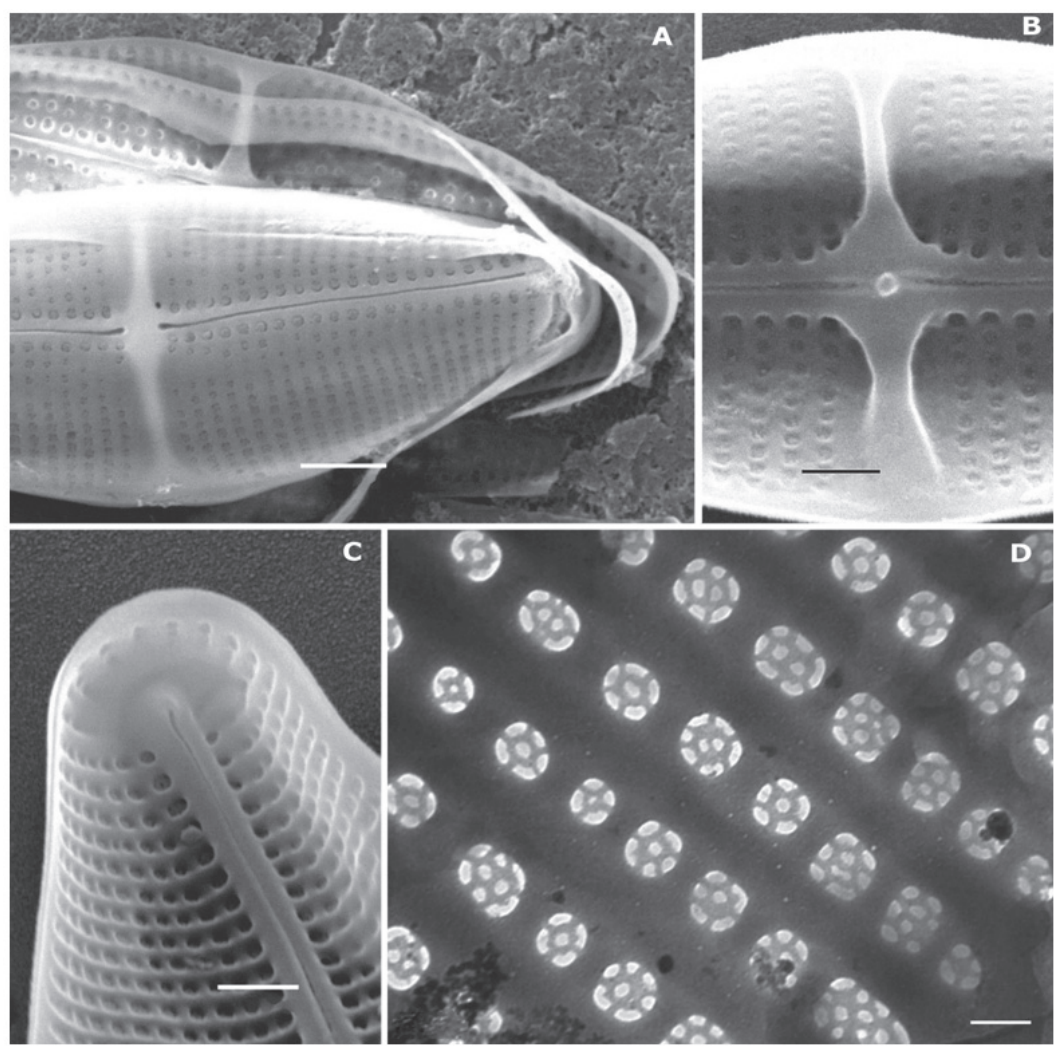

Figura 2. Craspedostauros indubitabilis. Figs. A-C SEM, Fig. D TEM. A. Aréolas de mayor tamaño al lado del rafe; vista externa de la curvatura de los extremos proximales del rafe; estauro y fascia. B. Vista interna del centro de la valva mostrando una protuberancia entre los extremos proximales del rafe; estauro más angosto que la fascia. C. Vista interna de valva con extremo redondeado. D. Cribra de aréolas con número variable de poros. Escalas: $\mathrm{A}=2 \mu \mathrm{m}, \mathrm{B}-\mathrm{C}=1 \mu \mathrm{m}, \mathrm{D}=0,2 \mu \mathrm{m}$.

Figure 2. Craspedostauros indubitabilis. Figs. A-C SEM, Fig. D TEM. A. Areolae adjacent to the raphe larger than the rest; external view of central raphe fissures, stauros and fascia. B. Internal view of central part of a valve showing the knob-like thickening between the proximal raphe ends; stauros narrower than the fascia. C. Internal view of a valve with a rounded apice. D. Areolae cribrate with a variable number of pores. Scale bars: $\mathrm{A}=2 \mu \mathrm{m}, \mathrm{B}-\mathrm{C}=1 \mu \mathrm{m}, \mathrm{D}=0.2 \mu \mathrm{m}$.

Ocho especies han sido asignadas al género Craspedostauros, cuyas principales características morfológicas se resumen en la Tabla I. C. neoconstrictus (Cox 1999a) presenta un eje apical de hasta $110 \mu \mathrm{m}$ de largo, las aréolas tienen alrededor de 6 poros en cada cribra y presenta engrosamientos tipo helictoglosa en las fisuras centrales internas del rafe. C. indubitabilis es, en general, de menor tamaño, el número de poros en la cribra, como ya se ha mencionado, varía entre 3 y $13(2 / 1-9 / 4)$, y presenta una protuberancia redondeada entre los extremos centrales internos del rafe. C. amphoroides y C. decipiens (Cox 1999a) tienen valvas de contorno lanceolado, existen 5-13 poros en cada cribra y carecen de una protuberancia entre los extremos proximales internos del rafe, característica presente y muy constante en $C$. indubitabilis, la que, además, presenta valvas linear-elípticas, muy raramente lanceoladas. En C. australis (Cox 1999a) el número de estrías sobre la valva, cerca de 35 en $10 \mu \mathrm{m}$, es superior al presente en $C$. indubitabilis $(22-30$ en $10 \mu \mathrm{m}) \mathrm{y}$ existen solamente 4 poros en cada cribra, 4/0 (Cox 1999a, b). C. britannicus tiene solamente 24 estrías de aréolas en $10 \mu \mathrm{m}$,
4 o más poros en cada cribra $(4 / 0$ - 4/1) y doble helictoglosa interna en los extremos centrales del rafe. C. cappensis (Cox 1999a) difiere de C. indubitabilis por el contorno lanceolado de sus valvas, por el menor número de estrías sobre la valva (alrededor de 19 en $10 \mu \mathrm{m}$ ) y por presentar 5 -16 poros en cada cribra (5/6 - 8/8); sin embargo, al igual que $C$. indubitabilis, tiene una protuberancia central entre los extremos internos del rafe. Finalmente, C. laevissimus (Sabbe et al. 2003) es la especie más cercana a $C$. indubitabilis. Este taxón, descrito para la Antártida por West \& West (1911) como Tropidoneis laevissima W. West \& G.S. West, comparte muchas de las características presentes en $C$. indubitabilis, difiriendo de ella en la orientación radial que a veces presentan las estrías en el centro de la valva, por el contorno circular de las aréolas, con un menor número de poros en cada cribra (generalmente 4/1), y por la carencia de una protuberancia central entre los extremos centrales internos del rafe, que terminan en una doble helictoglosa. Además, según Sabbe et al. (2003) sería la única especie del género que se desarrolla exclusivamente en lagos salinos y no en el litoral marino. 


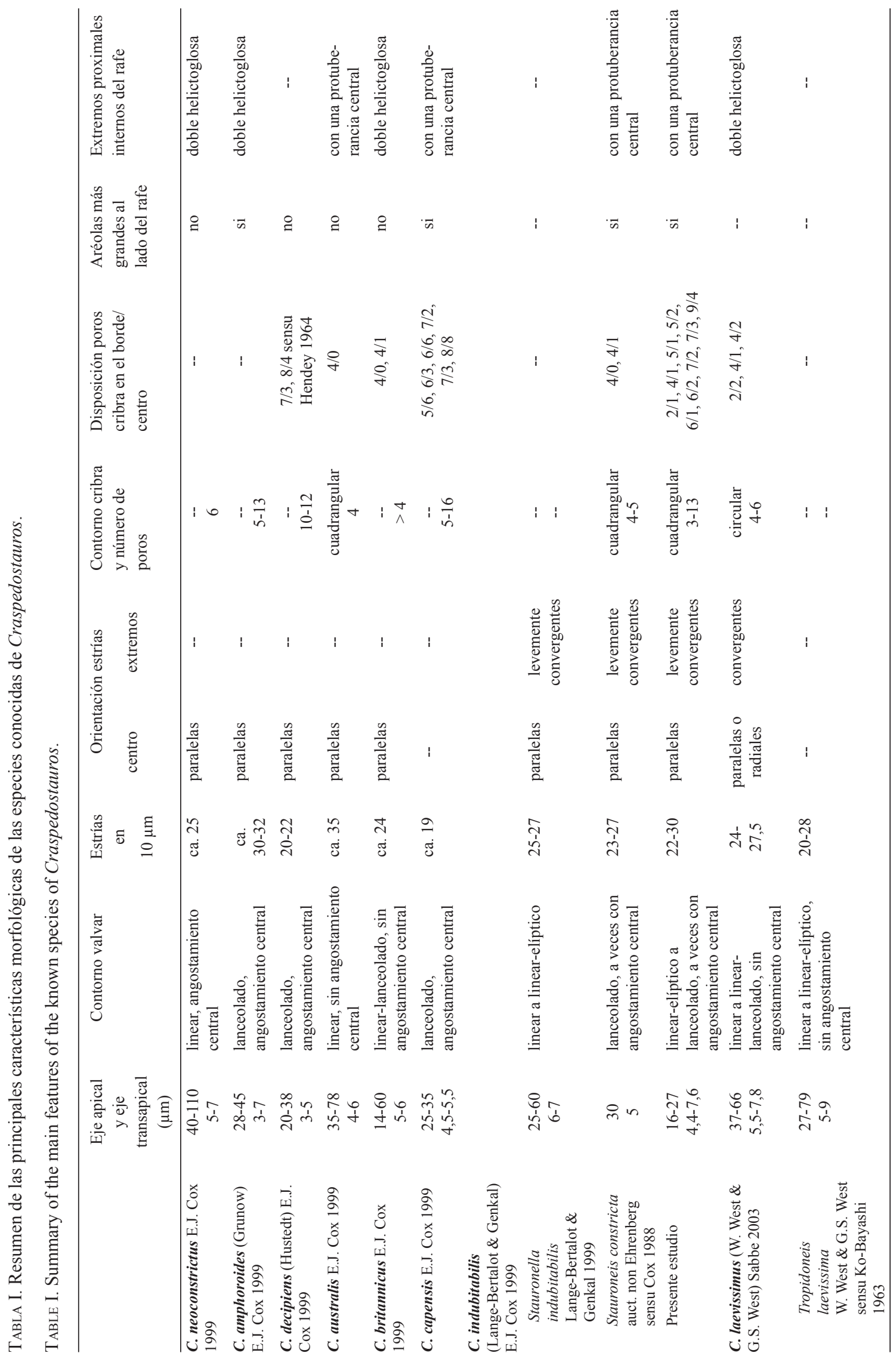


Craspedostauros indubitabilis es una especie marina, propia de aguas salobres y costeras, y también está presente en lagos salinos. Descrita para el Océano Ártico como Stauronella indubitabilis, ha sido señalada para diversas localidades en el Hemisferio Norte (Witkowski et al. 2000, Ulanova \& Snoeijs 2006), en lagos de la Antártida (Spaulding et al. 1997), y en las Islas Sandwich del Sur (Convey et al. 2000). Por otra parte, C. laevissimus fue descrita como una especie endémica de la Antártida, en lagos con alta salinidad (Fukushima 1962, 1964, Watanuki 1979, Priddle \& Belcher 1981, Roberts \& McMinn 1999, Sabbe et al. 2003).

\section{AGRADECIMIENTOS}

Agradecemos los valiosos comentarios y sugerencias del Dr. Eduardo Morales (Universidad Católica Boliviana San Pablo, Bolivia) y de dos evaluadores anónimos. Agradecemos también la ayuda otorgada por la Dirección de Investigación Científica de la Universidad de Concepción, Chile, y la asistencia del personal del Laboratorio de Microscopía Electrónica y del Laboratorio de Microalgas del Departamento de Botánica, ambos de la mencionada Universidad. Este estudio fue financiado parcialmente por el Proyecto FONDEF DO71-1017, "Biotechnology applied to production of food and balanced diets for improving the North Scallop culture".

\section{BIBLIOGRAFÍA}

Anonymous. 1975. Proposals for standardization of diatom terminology and diagnosis. Nova Hedwigia Beiheft 53: 323-354.

Convey, P., I. Lewis Smith, D.A. Hodgson \& H.J. Peat. 2000. The flora of the South Sandwich Islands, with particular reference to the influence of geothermal heating. Journal of Biogeography 27: 1279-1295.

Cox, E.J. 1988. Taxonomic studies on the Diatom Genus Navicula. V. The establishment of Paralibellus gen. nov. for some members of Navicula sect. Microstigmaticae. Diatom Research 3: 9-38.

Cox, E.J. 1999a. Craspedostauros gen. nov., a new diatom genus for some unusual marine raphid species previously placed in Stauroneis Ehrenberg and Stauronella Mereschkowsky. European Journal of Phycology 34: 131-147.

Cox, E.J. 1999b. Variation in patterns of valve morphogenesis between representatives of six biraphid diatoms genera (Bacillariophyceae). Journal of Phycology 35: 1297-1312.

Cox, E.J. \& D.M. Williams. 2000. Systematics of naviculoid diatoms: the interrelationship of some taxa with a stauros. European Journal of Phycology 35: 273-282.

EhrenBerG, C.G. 1843. Verbreitung und Einfluss des mikroskopischen Lebens in Süd-und Nord-Amerika.
Abhandlungen der Königlichen Akademie der Wissenschaften zu Berlin 1841: 291-445, 4 pls.

Fukushima, H. 1962. Diatoms from the Shin-Nan Rock Ice-Free Area, Prince Olav Coast, the Antarctic Continent. Antarctic Record 14: 80-91.

Fukushima, H. 1964. Diatoms vegetation on ice-free area of Cape Royds, Antarctica. Antarctic Record 22: 1-13.

Hasle, G.R. \& G.A. Fryxell. 1970. Diatoms: cleaning and mounting for light and electron microscopy. Transactions of the American Microscopical Society 89: 469-474.

HENDEY, N.I. 1964. An Introductory Account of the smaller Algae of British Coastal Waters. Part V: Bacillariophyceae (Diatoms). Her Majesty's Stationery Office, London. 317 pp, 45 pls.

Ko-Bayashi, T. 1963. Variations on some pennate diatoms from Antarctica. Japanese Antarctic Research Expedition 19561962. Scientific Reports, Series E 18: 1-20.

Lange-Bertalot, H. \& S.I. Genkal. 1999. Diatomeen aus Sibirien I. Inseln im Arktischen Ozean (Yugorsky-Shar Strait). Iconographia Diatomologica 6:1-271.

MereschKowsky, C. 1901. On Stauronella, a new genus of diatoms. Annals and Magazine of Natural History, Series 7, 8: 424434.

Priddle, J. \& J.H. Belcher. 1981. Freshwater biology at Rothera Point, Adelaide Island II. Algae. British Antarctic Survey Bulletin 53: 1-9.

Roberts, D. \& A. McMinn. 1999. Diatoms of the saline lakes of the Vestfold Hills, Antarctica. Bibliotheca Diatomologica 44: 1-83.

Ross, R., E.J. Cox, N.I. Karayeva, D.G. Mann, T.B.B. Paddock, R. Simonsen \& P.A. Sims.1979. An amended terminology for the siliceous components of the diatom cell. Nova Hedwigia Beiheft 64: 513-533.

Sabbe, K., E. Verleyen, D.A. Hodgson, K. Vanhoutte \& W. VyVERMAN. 2003. Benthic diatom flora of freshwater and saline lakes in the Larsemann Hills and Rauer Islands, East Antarctica. Antarctic Science 15: 227-248.

Spaulding, S.A., D.M. McKnight, E.F. Stoermer, \& P.T. Doran. 1997. Diatoms in sediments of perennially ice-covered Lake Hoare, and implications for interpreting lake history in the dry valleys region of Antarctica. Journal of Paleolimnology 17: 403-420.

Ulanova, A. \& P. Snoeiss. 2006. Gradient responses of epilithic diatom communities in the Baltic Sea proper. Estuarine, Coastal and Shelf Science 68: 661-674.

Walne, R.R. 1970. Studies on food value of nineteen genera of algae to juvenile bivalves of the genera Ostrea, Crassostrea, Mercenaria and Mytilus. Ministry of Agriculture, Fisheries and Food, Series II 26: 1-45.

WatANUKI, T. 1979. Isolation and culture of Antarctic diatoms from the saline lakes in the Soya Coast, East Antarctica. Memoirs of National Institute of Polar Special Research Issue 11: 35-41.

West, W. \& G.S. West. 1911. Freshwater Algae. British Antarctic Expedition 1907-9, under the command of Sir E.H. Shackleton, C.V.O., Biology. Part VII, Vol. 1, London. pp. 263-298, pls. 24-26.

Witkowski, A., H. Lange-Bertalot \& D. Metzeltin. 2000. Diatom Flora of Marine Coast I. Iconographia Diatomologica 7: $1-925$.

Recibido: 15.04 .11

Aceptado: 13.05.11 